\title{
Perceptual and Semantic Components of Memory for Objects and Faces: A PET Study
}

\author{
Jon S. Simons ${ }^{1}$, Kim S. Graham ${ }^{1}$, Adrian M. Owen ${ }^{1,2}$, \\ Karalyn Patterson ${ }^{1}$, and John R. Hodges ${ }^{1,3}$
}

\begin{abstract}
Previous studies have suggested differences in the neural substrates of recognition memory when the contributions of perceptual and semantic information are manipulated. In a within-subjects design PET study, we investigated the neural correlates of the following factors: material type (objects or faces), semantic knowledge (familiar or unfamiliar items), and perceptual similarity at study and test (identical or different pictures). There was consistent material-specific lateralization in frontal and temporal lobe regions when the retrieval of different types of nonverbal stimuli was compared, with objects
\end{abstract}

\section{INTRODUCTION}

Neuropsychological and functional imaging investigations have greatly advanced our understanding of human long-term memory in recent years. Interest among cognitive neuroscientists has traditionally focused on the important role of medial temporal (Tulving \& Markowitsch, 1998; Vargha-Khadem et al., 1997; Cohen \& Eichenbaum, 1993; Squire, 1987, 1992) and frontal (Moscovitch \& Winocur, 1995; Shimamura, 1995; Wheeler, Stuss, \& Tulving, 1995; Milner, Petrides, \& Smith, 1985) lobe structures in memory processes. Although several early neuroimaging studies failed to find memory-related activation in the medial temporal lobe, more recent studies have documented activity differences in this region during the encoding and retrieval of new information (Schacter \& Wagner, 1999; Schacter et al., 1997; Kelley et al., 1998; Dolan \& Fletcher, 1997; Gabrieli, Brewer, Desmond, \& Glover, 1997; Owen, Milner, Petrides, \& Evans, 1996; Tulving, Markowitsch, Craik, Habib, \& Houle, 1996).

The importance of areas in the prefrontal cortex for long-term memory has also been highlighted by functional imaging studies (McDermott, Buckner, Petersen, Kelley, \& Sanders, 1999; Buckner \& Koutstaal, 1998; Buckner, Kelley, \& Petersen, 1999; Fletcher, Shallice, \&

\footnotetext{
${ }^{1}$ MRC Cognition and Brain Sciences Unit, Cambridge, UK,

${ }^{2}$ Wolfson Brain Imaging Centre, Addenbrooke's Hospital, Cambridge, UK, ${ }^{3}$ University Neurology Unit, Addenbrooke's Hospital, Cambridge, UK
}

activating bilateral areas and faces preferentially activating the right hemisphere. Retrieval of memories for nameable, familiar items was associated with increased activation in the left ventrolateral prefrontal cortex, while memory for unfamiliar items involved occipital regions. Recognition memory for different pictures of the same item at study and test produced blood flow increase in left inferior temporal cortex. These results have implications for our understanding of the neural correlates of perceptual and semantic contributions to recognition memory.
Dolan, 1998; Fletcher, Shallice, Frith, Frackowiak, \& Dolan, 1998; Kelley et al., 1998; Dolan \& Fletcher, 1997). Evidence has accumulated to support the idea that the left and right frontal cortex are lateralized for encoding and retrieval of memories (Fletcher, Shallice, \& Dolan, 1998; Fletcher, Shallice, Frith, et al., 1998; Dolan \& Fletcher, 1997; Nyberg, Cabeza, \& Tulving, 1996; Shallice et al., 1994; Tulving, Kapur, Craik, Moscovitch, \& Houle, 1994). The results of some more recent studies, however, suggest that both left and right prefrontal areas are involved in both episodic encoding and retrieval, with the relative lateralization of activation depending instead upon the type of material being remembered (Lee, Robbins, Pickard, \& Owen, 2000; Kim et al., 1999; McDermott et al., 1999; Kelley et al., 1998; Klingberg \& Roland, 1998; Wagner, Desmond, Glover, \& Gabrieli, 1998; Wagner, Poldrack, et al., 1998).

\section{Material-Specific Lateralization}

Much recent interest in the neuroimaging literature has centered on the idea that memory for different types of material may be lateralized to different regions within the left and right hemispheres. Although such lateralization is an established concept in the neuropsychological literature (Baxendale, 1997; Warrington, 1984; Milner, 1970, 1972; Warrington \& James, 1967; Gazzaniga, Bogen, \& Sperry, 1962), it is only recently that functional imaging studies of memory have begun to explore regional activations associated with the verbal/nonverbal 
nature of the stimuli employed (Kim et al., 1999; McDermott et al., 1999; Kelley et al., 1998; Wagner, Poldrack, et al., 1998). Of the few studies that have directly compared the encoding of verbal and nonverbal material, the majority has demonstrated that verbal encoding involves prefrontal and medial temporal regions in the left hemisphere and nonverbal encoding analogous regions in the right hemisphere (McDermott et al., 1999; Kelley et al., 1998; Wagner, Poldrack, et al., 1998). This same verbal/nonverbal lateralization has also been identified in memory retrieval studies (Lee et al., 2000; Kim et al., 1999; McDermott et al., 1999; Wagner, Poldrack, et al., 1998).

The term "nonverbal" covers many different types of stimuli, such as faces, textures, and objects. The characteristic pattern of activation in right hemisphere regions has been demonstrated when faces (Kim et al., 1999; McDermott et al., 1999; Kelley et al., 1998) and textures (Wagner, Poldrack, et al., 1998) are contrasted with words, but only one study has directly compared memory for objects and words within the same participants. Kelley et al. (1998) investigated the encoding of object, face, and word memories and found that, while faces and words activated the expected right and left prefrontal and medial temporal regions respectively, object encoding activated these regions bilaterally. The authors interpreted this result in terms of dual-coding theory (Paivio \& Csapo, 1973), suggesting that the encoding of nameable objects can draw upon both verbal and nonverbal information, hence the involvement of both hemispheres.

There are, to the best of our knowledge, no published studies reporting activations associated with the retrieval of different types of "nonverbal" material. The first aim of the present study, therefore, was to compare the retrieval of faces and objects within the same participants. Based on the findings described above, we hypothesized that the retrieval phase of recognition memory for faces compared with objects would activate predominantly right hemisphere regions (Kim et al., 1999; McDermott et al., 1999) within the prefrontal cortex and temporal lobe. Retrieval of memory for objects relative to faces, however, was expected to produce a bilateral pattern of activation in these areas, similar to that reported for related studies of encoding (Kelley et al., 1998).

\section{Manipulating Semantic Knowledge}

It has been argued that the bilateral pattern of activation produced when nameable objects are remembered reflects the involvement of both verbal (i.e., left hemisphere) and nonverbal (i.e., right hemisphere) processes. If true, then one might reasonably expect unfamiliar (i.e., novel) and, therefore, unnameable objects to produce a pattern of activation that is more strongly lateralized to the right hemisphere. On the same grounds, while memory for unfamiliar faces produces activations that are predominantly right-sided (Kim et al., 1999; McDermott et al., 1999; Kelley et al., 1998), familiar faces, which may be explicitly or implicitly named in the course of processing, may yield a less strongly lateralized pattern of activation.

In the only memory study of which we are aware that directly compared encoding of familiar and unfamiliar faces, encoding of famous faces did indeed produce bilateral activation (Kelley et al., 1999). A similar pattern was also reported recently in a comparison between a fame judgement task using famous faces and recognition memory for recently encoded unfamiliar faces (Leveroni et al., 2000). The second aim of the present study was to provide a direct comparison of recognition memory retrieval of famous and unfamiliar faces, and of nameable and novel objects, within the same participants. We hypothesized that compared to unfamiliar items, the additional verbal processing possible with familiar items might produce activation involving left prefrontal cortex areas implicated in the strategic processing of semantic information, and perhaps in the left temporal lobe, thought to be associated with semantic representations (Mummery et al., 1999; Wagner, Poldrack, et al., 1998; Dolan \& Fletcher, 1997; Thompson-Schill, D'Esposito, Aguirre, \& Farah, 1997; Vandenberghe, Price, Wise, Josephs, \& Frackowiak, 1996). Conversely, compared to familiar items, the predominantly nonverbal, perceptual processing required for recognition memory of unfamiliar items should be associated with more selective right hemisphere activation, perhaps including more posterior, visuoperceptual areas in the occipital lobes.

\section{Perceptual Similarity at Study and Test}

When familiar items are used as stimuli, both perceptual and semantic factors may contribute to successful recognition memory (Graham, Simons, Pratt, Patterson, \& Hodges, 2000; Bruce, 1982; Paivio \& Csapo, 1973). Indeed, it has been argued that the slight decrement in performance observed when different pictures of objects or faces are used at study and test (Srinivas, 1995; Cooper, Schacter, Ballesteros, \& Moore, 1992; Bruce, 1982) may reflect the fact that the value of the perceptual information available from seeing the target item in the study phase is relatively reduced. The decision as to whether the item was seen previously becomes more reliant on the semantic knowledge activated in common by the two pictures. If, as in Bruce's (1982) experiment, unfamiliar faces are used as stimuli, semantic knowledge contributes far less and a reduction in recognition memory performance may result.

Very few neuroimaging studies have investigated the effect of perceptual similarity of study and test stimuli on recognition memory, and only one has directly 
compared recognition memory for identical and differ-

Schacter et al. (1997) found greater activation in the ent pictures in the same participants. In that study, left hippocampus when the identical picture of a novel

Table 1. Significant Changes in Regional Cerebral Blood Flow in the Comparisons Between Recognition Memory for Objects and Faces (Threshold $=p<.05$, Corrected for Whole Brain Volume)

\begin{tabular}{|c|c|c|c|c|c|}
\hline \multirow[b]{2}{*}{ Brain Region } & \multicolumn{3}{|c|}{ Coordinates } & \multirow[b]{2}{*}{$t$} & \multirow[b]{2}{*}{$p$} \\
\hline & $x$ & $y$ & $z$ & & \\
\hline \multicolumn{6}{|c|}{ All Object Conditions minus All Face Conditions } \\
\hline \multicolumn{6}{|l|}{ Left hemisphere } \\
\hline Anterior prefrontal cortex (BA 10) & -40 & 45 & -2 & 5.78 & .002 \\
\hline Inferior parietal lobe (BA 40) & -48 & -47 & 39 & 4.90 & .044 \\
\hline \multirow[t]{2}{*}{ Inferior temporal gyrus (BA 37) } & -51 & -63 & -7 & 8.56 & .000 \\
\hline & -28 & -60 & -5 & 6.78 & .000 \\
\hline Inferior occipital gyrus/ fusiform (BA 18) & -34 & -87 & 8 & 9.00 & .000 \\
\hline \multicolumn{6}{|l|}{ Right hemisphere } \\
\hline Middle temporal gyrus (BA 37) & 61 & -57 & -7 & 5.20 & .016 \\
\hline Superior parietal lobe $(\mathrm{BA} 7 / 19)$ & 30 & -72 & 40 & 5.07 & .025 \\
\hline Middle occipital gyrus (BA 18) & 36 & -83 & 15 & 8.54 & .000 \\
\hline
\end{tabular}

All Face Conditions minus All Object Conditions

Left hemisphere

Dorsolateral prefrontal cortex (BA 9)

Uncus (BA 34/28)

Middle temporal gyrus (BA 21)

Cingulate gyrus (BA 23)

$\begin{array}{rrrrr}-4 & 58 & 25 & 4.9 & .044 \\ -28 & 9 & -24 & 5.17 & .018 \\ -40 & 12 & -29 & 4.98 & .034 \\ -2 & -57 & 21 & 6.44 & .000\end{array}$

Right hemisphere

Anterior prefrontal cortex (BA 11)

Temporal pole (BA 38)

Inferior temporal gyrus (BA 20)

Parahippocampal gyrus (BA 27/35)

Fusiform gyrus (BA 37)

Midline

Medial orbital gyrus (BA 10)

$\begin{array}{rrrrr}2 & 21 & -8 & 4.92 & .041 \\ 32 & 16 & -26 & 5.30 & .011 \\ 53 & -9 & -18 & 6.92 & .000 \\ 44 & -8 & -35 & 6.11 & .001 \\ 12 & -31 & -3 & 5.40 & .008 \\ 42 & -55 & -17 & 8.36 & .000 \\ & & & & \\ 0 & 46 & -12 & 6.34 & .000\end{array}$

Unfamiliar Objects minus Unfamiliar Faces

Left hemisphere

Posterior fusiform gyrus (BA 19)

$\begin{array}{rrrrr}-48 & -64 & -7 & 5.55 & .005 \\ -26 & -64 & -5 & 5.13 & .020 \\ -32 & -85 & 6 & 7.42 & .000\end{array}$

Fusiform/inferior occipital gyrus (BA 18)

$\begin{array}{lllll}-32 & -85 & 6 & 7.42 & .000\end{array}$

Right hemisphere

Middle occipital gyrus (BA 18)

$\begin{array}{lllll}34 & -83 & 15 & 8.62 & .000\end{array}$ 
Table 1. Continued

\begin{tabular}{|c|c|c|c|c|c|}
\hline \multirow[b]{2}{*}{ Brain Region } & \multicolumn{3}{|c|}{ Coordinates } & & \\
\hline & $x$ & $y$ & $z$ & $t$ & $p$ \\
\hline
\end{tabular}

Unfamiliar Faces minus Unfamiliar Objects

Right hemisphere

Inferior temporal gyrus (BA 20)

$\begin{array}{lllll}53 & -11 & -18 & 4.80 & .060\end{array}$

Familiar Objects Same minus Familiar Faces Same

No suprathreshold activations

Familiar Faces Same minus Familiar Objects Same

Right hemisphere

Lingual gyrus (BA 18)

$\begin{array}{lllll}6 & -82 & -6 & 5.14 & .020\end{array}$

object was presented at study and test than when either different orientations or different sizes of the objects were seen. In the opposite contrast (Orientation change - Identical object), activation was seen in the right temporal lobe. The authors concluded that the left hippocampus is sensitive to the physical similarity between novel objects seen at study and test. Because novel objects are "nonverbal" in that they cannot be easily named, the right temporal lobe activation seen when physical similarity was reduced might reflect the greater demands on right hemisphere systems involved in the processing of perceptual characteristics (in the absence of a contribution from semantic knowledge) in this condition.

It is, therefore, of great interest to explore the effect of physical similarity when nameable objects and faces (which can draw upon semantic knowledge) are used as stimuli. Addressing this issue was the third aim of the present study. Assuming that the findings of Schacter et al. (1997) can be transferred to familiar items, it was predicted that recognition memory for identical pictures, relative to different pictures of the same item, would be associated with greater activation in the left hippocampus. In the opposite comparison, however, it was expected that recognition memory would become more reliant upon semantic knowledge about the target item in order to compare the representations activated by perceptually different pictures. We predicted, therefore, that blood flow increase would be seen in the region of the left inferolateral temporal lobe (Mummery et al., 1999; Vandenberghe et al., 1996).

\section{Predicted Patterns of Activation}

This study was designed to examine the activation differences observed when perceptual and semantic manipulations are applied to a recognition memory task. To summarize, the predictions were that recognition memory retrieval of faces relative to objects would preferentially activate prefrontal and temporal regions in the right hemisphere, but retrieval of objects compared to faces would produce a bilateral pattern of activation in these areas. Memory for familiar over unfamiliar items would activate the left prefrontal and temporal cortex, while the opposite contrast would be associated with activation in occipital lobe areas. Identical pictures at study and test would lead to increased blood flow in the left hippocampus, relative to the use of different exemplars at study and test. The opposite comparison would cause activation centered on the left temporal lobe.

\section{RESULTS}

\section{Behavioral Results}

Behavioral data for three participants were lost due to equipment failure. For the remaining participants, hit (and false alarm) rates for each condition were: familiar objects same (FOS) $=0.93(0.00)$, familiar faces same $(\mathrm{FFS})=0.94(0.02)$, familiar objects different $(\mathrm{FOD})=$ 0.80 (0.07), familiar faces different (FFD) $=0.92(0.03)$, unfamiliar objects (UFO) $=0.87$ (0.12), and unfamiliar faces $(\mathrm{UFF})=0.88(0.08)$. As would be expected based on previous studies that have used similar stimulus manipulations (e.g., Kelley et al., 1998; Schacter et al., 1997), a significant effect of condition on recognition memory accuracy $\left(P_{\text {hits }}-P_{\text {false alarms }}\right)$ was observed $[F(5,25)=3.5, p<.05]$. Analysis of relevant post hoc comparisons revealed this to be attributable to accuracy differences between FOS and FOD $[t(5)=2.7, p<.05]$, FOS and UFO $[t(5)=2.6, p<.05]$, and FFS and UFF $[t(5)=3.5, p<.05]$, although none of these differences 


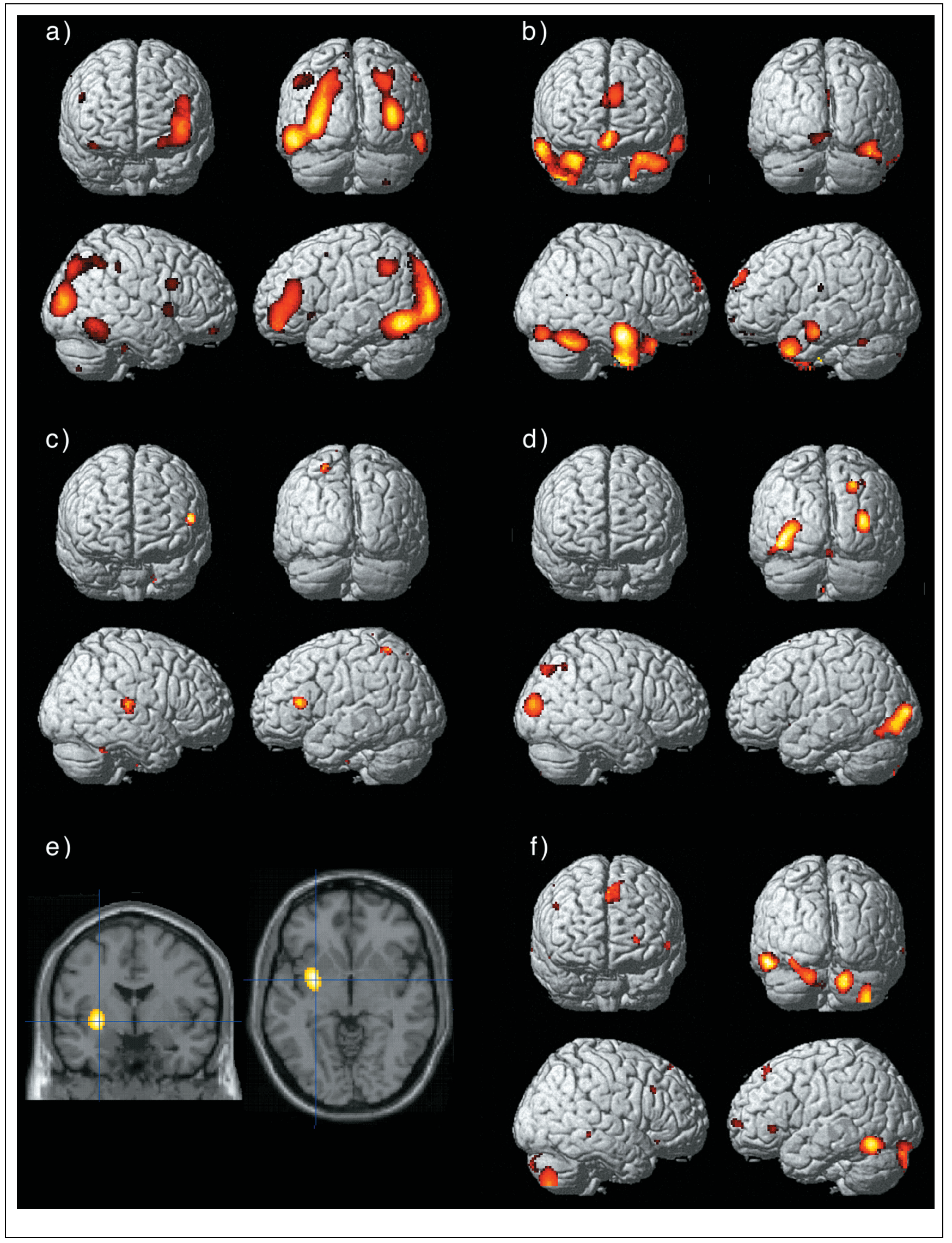


Table 2. Significant Changes in Regional Cerebral Blood Flow in the Comparisons Between Recognition Memory for Familiar and Unfamiliar Stimuli (Threshold $=p<.05$, Corrected for Whole Brain Volume)

\begin{tabular}{|c|c|c|c|c|c|}
\hline \multirow[b]{2}{*}{ Brain Region } & \multicolumn{3}{|c|}{ Coordinates } & \multirow[b]{2}{*}{$t$} & \\
\hline & $x$ & $\mathrm{y}$ & $\mathrm{Z}$ & & $p$ \\
\hline
\end{tabular}

Familiar Item Conditions minus Unfamiliar Item Conditions

Left hemisphere

Ventrolateral prefrontal cortex (BA 45)

$\begin{array}{lllll}-50 & 26 & 10 & 4.02 \quad .000 *\end{array}$

Unfamiliar Item Conditions minus Familiar Item Conditions

No suprathreshold activations

Unfamiliar Objects minus Familiar Objects Same

Right hemisphere

Middle occipital gyrus (BA 18)

$\begin{array}{lllll}34 & -85 & 17 & 4.90 & .043\end{array}$

Familiar Objects Same minus Unfamiliar Objects

No suprathreshold activations

Unfamiliar Faces minus Familiar Faces Same

No suprathreshold activations

Familiar Faces Same minus Unfamiliar Faces

No suprathreshold activations

${ }^{*}$ Accepted at uncorrected level (see Methods).

was significant once corrections for multiple comparisons were made.

Following each experimental condition, participants were debriefed about the task they had just undertaken. This process confirmed that the familiar objects and faces were indeed well-known to the participants.

\section{PET Results}

\section{Objects versus Faces}

Comparison of the object conditions (FOS, FOD, UFO) and the face conditions (FFS, FFD, UFF) produced significant blood flow changes as shown in Table 1. The subtraction All objects - All faces produced bilateral patterns of activation (illustrated in Figure 1a) in the posterior part of the temporal lobes (BA 37), the parietal lobes (BA 40 on the left, BA 7/ 19 on the right) and inferior occipital lobes (BA 18). There was also activation in the left anterior prefrontal cortex (BA 10). The opposite subtraction (All faces - All objects) activated areas in the anterior temporal lobes bilaterally, although more extensively on the right (BA 21 on the left, BAs 38 and 20 on the right) (see Figure 1b). There were further activations in the region of the parahippocampal gyrus (BA 27/35) and

Figure 1. Regions of significant activation shown at an uncorrected threshold of $p<.001$ superimposed upon an average rendered brain (projections clockwise from top-left are: anterior, posterior, left, and right), or (e) upon an average T1-weighted MRI (coronal and horizontal projections). (a) All object conditions minus all face conditions: bilateral temporal, parietal, and occipital activations, also left prefrontal cortex, exceeded corrected threshold of $p<.05$ (b). All face conditions minus all object conditions: primarily right pattern of activations in temporal lobes, both laterally and medially, as well as in prefrontal cortex, exceeded corrected thershold. (c) Familiar item conditions minus unfamiliar item conditions: left ventrolateral prefrontal cortex exceeded uncorrected threshold of $p<.001$ (see Methods). (d) Unfamiliar objects minus familiar objects same: bilateral activation in occipital lobes, although only that on right reached corrected threshold of $p<.05$. (e) Same item conditions minus different item conditions: illustrates activation within left putamen significant at corrected threshold of $p<.05$. (f) Different item conditions minus item conditions: activations: activations exceeding corrected threshold of $p<.05$ are left posterior inferior temporal lobe and right cerebellum. 
the posterior fusiform gyrus (BA 37), both on the right, as well as in the anterior prefrontal cortex, both in the midline (BA 10) and on the right (BA 11). Other regions showing significant activations were the dorsolateral prefrontal cortex (BA 9), the uncus (BA 34/28), and the cingulate gyrus (BA 23), all on the left.

When the UFO and UFF conditions were compared (UFO - UFF), the same bilateral network of occipital lobe activations was found as in the All objects - All faces subtraction, involving the posterior fusiform gyrus (BAs 18 and 19) on the left and the middle occipital gyrus (BA 18) on the right. The opposite comparison (UFF - UFO) produced the same right anterior temporal lobe (BA 20) activation as in the All faces - All objects subtraction, which tended towards corrected significance $(p=.06)$. When the two familiar same conditions were contrasted, FOS FFS revealed no activations that survived correction, but the opposite subtraction (FFS - FOS) was asso- ciated with activation of the right inferior surface of the occipital lobe (BA 18).

\section{Familiar versus Unfamiliar}

Table 2 shows the areas of significant blood flow change when two of the familiar conditions (FOS and FFS) were compared with both of the unfamiliar conditions (UFO and UFF). The subtraction Familiar items - Unfamiliar items produced significant activation (see Figure 1c) in the left ventrolateral prefrontal cortex (BA 45). No activations survived the corrected threshold in the opposite subtraction (Unfamiliar items - Familiar items). When unfamiliar and familiar objects were compared directly (UFO - FOS), increased blood flow was observed in the occipital lobes bilaterally (BA 18), although only the activation on the right reached corrected significance (see Figure 1d). The opposite subtraction (FOS - UFO) produced activation in the left temporal lobe (BA 37), although this failed to exceed the corrected threshold. Neither of the analogous face contrasts (UFF

Table 3. Significant Changes in Regional Cerebral Blood Flow When Recognition Memory For Items that are Identical at Study and Test is Compared with Recognition Memory When Different Pictures of Items are Used in the Study and Test Phases (Threshold $=$ $p<.05$, Corrected for Whole Brain Volume)

\begin{tabular}{|c|c|c|c|c|c|}
\hline \multirow[b]{2}{*}{ Brain Region } & \multicolumn{3}{|c|}{ Coordinates } & \multirow[b]{2}{*}{$t$} & \\
\hline & $x$ & $\mathrm{y}$ & z & & $p$ \\
\hline
\end{tabular}

Same Item Conditions minus Different Item Conditions

Left hemisphere

Putamen

$\begin{array}{llll}-28 & -2 & -3 & 5.12 .021\end{array}$

Different Item Conditions minus Same Item Conditions

Left hemisphere

Inferior temporal gyrus (BA 37)

$\begin{array}{cccc}-51 & -61 & -10 & 5.44 .007 \\ 16 & -79 & -25 & 5.51 .005\end{array}$

Cerebellum-Crus I

Familiar Objects Different minus Familiar Objects Same

Left hemisphere

Inferior temporal gyrus

(BA 37)

$\begin{array}{llll}-51 & -59 & -11 & 5.14 .019\end{array}$

Right hemisphere

Cerebellum-Crus I

Cerebellum-Crus VI

$\begin{array}{llll}36 & -76 & -38 & 5.31 .011 \\ 16 & -77 & -23 & 5.47 .006\end{array}$

Familiar Faces Different minus Familiar Faces Same

No suprathreshold activations 
- FFS, FFS - UFF) was associated with significant activations.

\section{Same versus Different}

The regions of activation when the same conditions (FOS and FFS) were contrasted with the different conditions (FOD and FFD) are presented in Table 3. In the subtraction Same items - Different items, significant activation was seen in the left putamen (see Figure 1e). The subtraction Different items - Same items was associated with significant activation in the left posterior temporal lobe (BA 37) and the right cerebellum (see Figure 1f).

For the objects conditions alone, the subtraction FOD - FOS activated the same region of the posterior temporal lobe (BA 37) on the left, and areas of the cerebellum on the right, as in the Different items - Same items contrast. The analogous subtraction using faces (FFD FFS), however, yielded no suprathreshold activations.

\section{DISCUSSION}

The present study has addressed three outstanding questions in the study of human long-term memory. Using a within-subjects design, the effects of three factors on recognition memory retrieval could be investigated directly, permitting more confidence in the interpretation of results than when conclusions are drawn from across different studies. Manipulation of material type (objects or faces), semantics (familiar or unfamiliar items), and perceptual similarity at study and test (identical or different pictures) resulted in blood flow differences that are consistent both with neuropsychological and functional imaging evidence, and that have implications for theories of human long-term memory.

When recognition memory for objects and faces was compared, consistent bilateral patterns of activation characterized memory for objects minus faces, whereas the opposite contrast involved regions in the right hemisphere more extensively. Recognition memory for familiar relative to unfamiliar items was associated with activation in the left ventrolateral prefrontal cortex, whereas the unfamiliar minus familiar comparison activated the occipital region, especially on the right. In conditions where items were the same at study and test, activation in the left putamen was observed, but when different pictures of the items were used in the study and test phases, significant activations were consistently found in the posterior left temporal lobe and right cerebellum.

\section{Material-Specific Lateralization}

The first of these results, regarding the retrieval of object and face memories, is of importance to the current debate about the lateralization of memory processes.
The present data provide further support for the view that it is the type of material being remembered (rather than the type of memory process being undertaken) that determines the relative lateralization of activation. Consistent with the one imaging study to date that has directly compared encoding of objects and faces (Kelley et al., 1998), and consistent with our predicted pattern of activation for retrieval, we found bilateral blood flow increases in the posterior temporal, parietal, and occipital lobes for objects relative to faces. Faces preferentially activated right hemisphere areas in the frontal, lateral and medial temporal, and occipital lobes, a predominantly right-lateralized pattern again similar to the findings of Kelley et al. Activation of right hemisphere regions has also been observed when faces (Kim et al., 1999; McDermott et al., 1999; Kelley et al., 1998) and textures (Wagner, Poldrack, et al., 1998) are contrasted with words, indicating that this predominant lateralization of activation is characteristic for material that emphasizes nonverbal, perceptual processing.

Although the present study was not designed to compare encoding and retrieval, the consistent patterns of activation seen here for retrieval add to accumulating evidence suggesting that left and right frontal lobes may not be asymmetrically involved in encoding and retrieval, as was previously thought (Nyberg, Cabeza, et al., 1996; Tulving et al., 1994). Instead, the present results support the view that the relative lateralization of activation depends upon the extent to which verbal or nonverbal processing is involved, rather than the type or phase of memory process. Material-specific lateralization in frontal and temporal regions is compatible with neuropsychological evidence. Patients with structural damage to the left temporal lobe, for example, often show disproportionate impairment on verbal memory tasks, whereas those with right hemisphere damage tend to perform poorly on memory tests involving nonverbal stimuli such as faces (Simons, Graham, Galton, Patterson, \& Hodges, 2001; Baxendale, 1997; Warrington, 1984; Milner, 1970, 1972).

\section{Manipulating Semantic Knowledge}

In line with the views discussed above, familiar items (which can draw upon verbal and/or semantic information) relative to unfamiliar items (which presumably rely to a greater extent upon nonverbal, perceptual processing) produced the predicted activation in the left ventrolateral prefrontal cortex, and a hint of involvement of the inferior temporal lobe (although this did not reach significance). The left ventrolateral prefrontal cortex has been implicated in relatively automatic strategic processes associated with semantic memory (Wagner, Poldrack, et al., 1998; Dolan \& Fletcher, 1997; Thompson-Schill et al., 1997), such as the selection and/or active retrieval of semantic knowl- 
edge that is assumed to be represented mainly in inferolateral temporal regions (Mummery et al., 1999; Vandenberghe et al., 1996).

Left ventrolateral prefrontal cortex activations have been observed in a variety of tasks in which semantic information is present, regardless of the level of processing involved (Murtha, Chertkow, Beauregard, \& Evans, 1999; Thompson-Schill et al., 1997). By contrast, blood flow increases in inferior temporal lobe areas have been associated with greater demands on semantic knowledge (Murtha et al., 1999). In the present study, we found no clear evidence that the inferior temporal lobe was activated in the comparison between familiar and unfamiliar items. These results are consistent with the view that in-depth processing of semantic information is typically not crucial for success in recognition memory. An exception to this general principle occurs when the recognition memory task is modified to place greater demands on the selection and retrieval of semantic representations, as in the manipulation of perceptual similarity at study and test (see below).

As predicted, there was evidence that memory for unfamiliar objects involved occipital lobe regions associated with perceptual processes (see Cabeza \& Nyberg, 2000 for a review). The contrast involving all the unfamiliar minus the familiar conditions failed to show significant activations, perhaps because of variability in the amount of perceptual and semantic information available in the different sets of stimuli even after efforts were made to reduce this variability (see Methods). Analysis of the object conditions separately, however, did reveal activation by novel objects of visuoperceptual areas in the occipital lobes, particularly on the right. This suggests that, in the absence of semantic information, recognition memory relies upon the processing of nonverbal, perceptual information, which involves primarily right hemisphere occipital regions. Such a view is consistent with behavioral studies involving patients with semantic dementia (Patterson \& Hodges, 2000; Hodges, Patterson, Oxbury, \& Funnell, 1992) who have a selective impairment to semantic knowledge associated with progressive atrophy of the temporal lobe. Patients who had severely disrupted semantic knowledge about an item could nevertheless recognize the item as having been seen previously in a recognition memory test. The basis for this successful episodic recognition, it was argued, is perceptual information from seeing the identical item at study (Graham et al., 2000; Simons, Graham, \& Hodges, 1999; Simons et al., 2001).

\section{Perceptual Similarity at Study and Test}

Unlike the study by Schacter et al. (1997) involving novel objects, we failed to find evidence that the use of identical pictures at study and test was associated with increased activation in the left hippocampus. Instead, we found significant activation within the left putamen. In the present study, we compared memory when identical nameable items were seen at study and test with that for different pictures of the items (e.g., a round-dial vs. a touch-button phone) in each phase. Schacter et al., however, contrasted different orientations or different sizes of the same novel objects. It is always problematic to interpret the lack of an activation, but it may be that these varying factors can explain the different results.

As predicted, the opposite comparison, of different minus same conditions, consistently produced activations involving the left inferolateral temporal lobe. This is in contrast to the right temporal lobe activations observed by Schacter et al. (1997) when comparing orientation change to identical picture in their novel object paradigm. The result obtained by Schacter et al. seems likely to reflect the fact that, in the absence of semantic information about the novel object seen, memory for differently orientated items relies upon right hemisphere systems involving the manipulation of perceptual representations of objects, perhaps mental rotation of the perceptual image until it matches an item stored in memory (Gerlach, Law, Gade, \& Paulson, 1999; Schacter et al., 1995).

In the present study, familiar, nameable items were used as stimuli in the conditions enabling the comparison between same and different pictures, meaning that semantic knowledge about the items could be used to support recognition memory. Remembering that a round-dial phone was seen at study could mean successful recognition memory of a touch-button phone because both share the same general object identity and name, "phone," irrespective of the fact that the items are not the same perceptually. Additionally, the different pictures used in the present study were perceptually different exemplars of the item, rather than just perceptual manipulations of the same picture. Memorial "strategies" like mental rotation would be of less use under these conditions than where the orientation of the same picture was changed between study and test. Taken as a whole, these results suggest that using different exemplars of familiar items at study and test increases demands upon semantic memory and/or naming, indicated by increased activation in the posterior left temporal lobe (Buckner, Koutstaal, Schacter, \& Rosen, 2000; Price, 1998).

Of note is that the activation peak found in the present study $(x=-51, y=-61, z=-10)$ for the Different minus Same picture contrast is almost identical to a region (peak at $x=-50, y=-58, z=$ -12) localized in a recent fMRI study of conceptual priming (Buckner et al., 2000). Buckner et al. (see also Price et al., 1996; Warburton et al., 1996) suggested that this region of inferior temporal cortex "may play a role in the formation, maintenance and/or interlink- 
ing of the conceptual lexical/semantic representations involved during task performance" (p. 637). The greater activation found in this region in the present study for different than same exemplars may, therefore, be associated with the selection and active retrieval of sufficient semantic information about items seen at study and test to support the decision as to whether they represent different exemplars of the same object or face. Alternatively or additionally, it has been suggested by both functional activation studies of normal individuals (Price, 1998) and by neuropsychological studies of aphasic patients (Foundas, Daniels, \& Vasterling, 1998) that this region may play a significant role in naming.

This evidence combines with recent proposals concerning the status of recognition memory in patients with semantic dementia to suggest that multiple inputs from perceptual and semantic systems contribute to recognition memory. In paradigms that employed different pictures of objects (Graham et al., 2000) and faces (Simons et al., 2001) at study and test, successful recognition memory by patients with semantic dementia was critically reliant upon conceptual knowledge about test items. The state of semantic knowledge was not a factor, however, when identical pictures were used at study and test. The authors argued that under normal circumstances (i.e., when there is perceptual equivalence between items used at study and test), perceptual information can be sufficient for successful recognition memory, but, when different pictures are used in the two phases, semantic information becomes critically important (Graham et al., 2000; Simons et al., 1999). The results of the present study and that by Schacter et al. (1997) provide neuroimaging evidence consistent with the multiple input hypothesis. The use of different pictures of familiar items at study and test forces the balance of reliance in recognition memory away from perceptual processing and towards recent activation of semantic information about the items seen at test. By contrast, a recognition memory paradigm employing unfamiliar, novel items that do not activate existing semantic representations shifts the balance of processing to the perceptual characteristics of the stimuli.

\section{CONCLUSION}

The present study has added to the currently small number of neuroimaging investigations that have used within-subjects methodology to address outstanding questions about the neural representation of memory for different types of stimulus material. In commonality with these other studies, we found that when recognition memory for objects and faces was compared directly, bilateral patterns of activation were observed for objects, while faces involved right hemisphere regions to a greater extent. Retrieval of memories for nameable, familiar items preferentially activated the left ventrolateral prefrontal cortex, an area associated with the strategic processing of semantic knowledge, while memory for unfamiliar items involved occipital visuoperceptual regions. The predicted hippocampal activation related to the use of identical stimuli at study and test was not observed, but when different pictures of items were seen at study and test, activation in left temporal lobe semantic memory regions was consistently found. Together, these results provide support for the view that recognition memory draws upon multiple inputs from perceptual and semantic processing systems. The results from the present study are consistent with the neuropsychological literature and, as such, provide a contribution to ongoing debates about the functional neuroanatomy of human long-term memory.

\section{METHODS \\ Participants}

Eleven males (mean age 25.4 years, range 22-31) were recruited primarily from the graduate student community at the University of Cambridge. All were righthanded with normal or corrected-to-normal vision, and had English as a first language. Participants were screened prior to arrival using a comprehensive medical questionnaire and all provided informed consent for participation.

\section{Design and Materials}

The three main aims of the study were addressed using six experimental conditions, which manipulated the factors objects/faces, familiar/unfamiliar, and same/different at study and test. We refer to the conditions as: familiar objects same (FOS), familiar faces same (FFS), familiar objects different (FOD), familiar faces different (FFD), unfamiliar objects (UFO), and unfamiliar faces (UFF). No baseline control condition was used because the study was designed to examine differences between memory conditions. Grayscale pictures or photographs, matched as closely as practicable for image size, contrast, brightness, and environmental cues, were used as stimuli in the six conditions. The images were primarily scanned from magazines and books, or downloaded from the Internet (see Figure 2 for examples of the stimuli used). Each of the conditions was administered twice during the scan session, with different stimuli used in each administration and the stimulus presentation order randomized for each participant. All of the stimuli used were extensively piloted outside the scanner to test for variations in task difficulty that might affect observed activations and to ensure that the everyday objects and famous faces were truly familiar.

In the two familiar objects conditions (FOS and FOD), 120 objects from nonliving categories such as household 
Figure 2. Examples of the stimuli used in the study and test phases of the six experimental conditions: familiar objects same, familiar objects different, familiar faces same, familiar faces different, unfamiliar objects, unfamiliar faces.

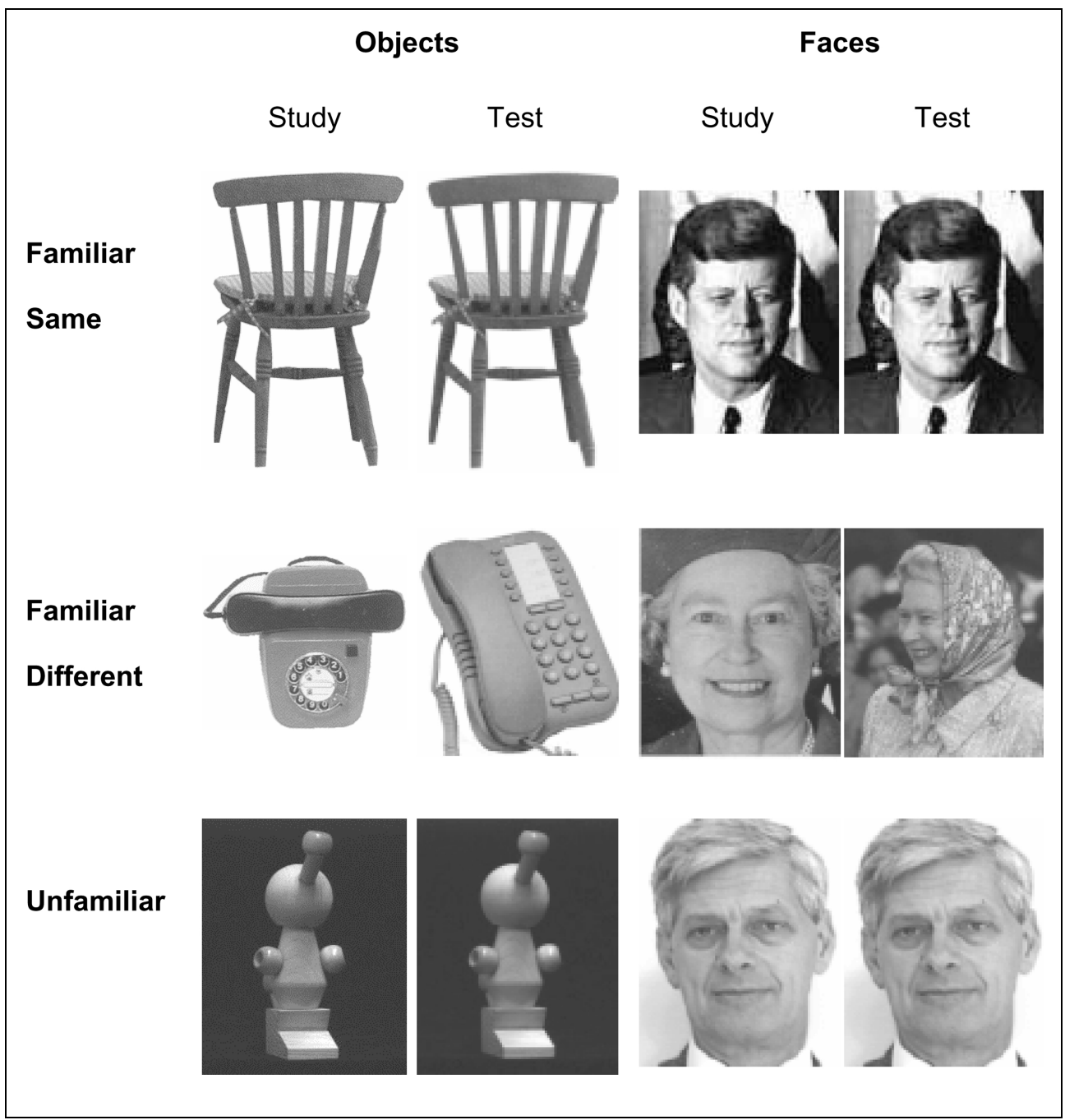

objects, vehicles, tools, and so on were used as stimuli (e.g., chair, helicopter, telephone). For 40 of the items, a different picture of the item was also located for use in the test phase of the FOD condition. Similarly, in the familiar faces conditions (FFS and FFD), 120 photographs of famous people (e.g., John F. Kennedy, Queen Elizabeth II, Audrey Hepburn) were used, and for 40 of the celebrities, a different photograph of the person was located for use in the FFD condition. Pilot testing suggested possible differences in the difficulty of the two face conditions, a factor that has been shown to result in significant blood flow variations (Nyberg, McIntosh, Houle, Nilsson, \& Tulving, 1996; Schacter, Alpert, Savage, Rauch, \& Albert, 1996). To mitigate against this concern, the celebrities used in the FFS condition were chosen to be of similar age and gender as each other, while these factors were varied in the FFD condition in order that the stimuli used were more easily distinguishable from one another.

The unfamiliar objects condition (UFO) utilized 60 pictures of novel or unfamiliar objects. Most of these comprised digitized pictures of novel wooden objects
(Srinivas, Breedin, Coslett, \& Saffran, 1997). These were interspersed with photographs of real but unfamiliar objects (electrical components, machinery parts, etc.). In the unfamiliar faces condition (UFF), 60 photographs of unfamiliar people were employed as stimuli. The photographs used were of male and female students and staff of varied age groups from a different university.

\section{Procedure}

Each participant was scanned 12 times, twice for each of the six experimental conditions. The presentation order of the conditions was counterbalanced across subjects and the order of the trials in each condition randomized for each participant. Stimuli were presented on an overhead monitor located where the participant could see it clearly. Behavioral responses were recorded using a button box. A portable Apple Mac G3 computer running PsyScope V1.2.4 (Cohen, MacWhinney, Flatt, \& Provost, 1993) was used to generate stimuli and collect behavioral data. When 
the participant was in the PET scanner but before the start of the scan session, the instructions were repeated and several practice trials undertaken for each of the experimental conditions, involving stimuli not used later in the experiment.

Fifty seconds before each PET data acquisition period, the participant was shown 20 pictures (each displayed for $2000 \mathrm{msec}$, with a 250-msec interstimulus interval) in a study phase and was asked to look at the pictures and to remember them. Test instructions were shown for 5 sec and then at the start of the acquisition sequence, recognition memory was tested for $45 \mathrm{sec}$. The participant was shown 20 more pictures (again displayed for 2000 msec each), half of which were items that had been seen in the study phase, half of which were novel foils. Participants pressed the left button on the button box if they thought an item had been seen at study and the right button if not. The instructions were varied slightly in the two Different conditions (FOD and FFD), with participants asked to press the left button if a different picture of the item had been seen at study and the right button if the item had not been seen.

Following each test phase, participants were immediately given a switch task lasting a further $45 \mathrm{sec}$. The switch technique (described by Cherry, Woods, Doshi, Banerjee, \& Mazziotta, 1995) is a method used to improve the signal-to-noise ratio by taking advantage of variations in arterial radioactivity concentration over the acquisition period. By administering the task of interest for the first half of the acquisition and then immediately switching to a task with different cognitive demands, improvements in signal-to-noise ratio of up to $30 \%$ have been found over the conventional method (Cherry et al., 1995) and the switch technique has been employed in a number of recent PET studies (e.g., Gerlach et al., 1999; Kanwisher, Woods, Iacoboni, \& Mazziotta, 1997). In the present experiment, the switch task comprised 20 trials in which participants viewed a square and had to press the left button if the square contained a large black dot and the right button if the square was empty.

\section{Image Acquisition and Data Analysis}

Twelve PET images were obtained for each participant using the GE Advance system, which produces 35 image slices at an intrinsic resolution of approximately $4.0 \times$ $5.0 \times 4.5 \mathrm{~mm}$. Participants received a 20-sec intravenous bolus of $\mathrm{H}_{2}{ }^{15} \mathrm{O}$ through a forearm cannula at a concentration of $300 \mathrm{MBq} \mathrm{ml}^{-1}$ and a flow rate of $10 \mathrm{ml} \mathrm{min}^{-1}$. With this method, each scan provides an image of rCBF integrated over a period of $90 \mathrm{sec}$ from when the tracer first enters the cerebral circulation.

The 12 scans were realigned using the first scan as a reference, normalized for global CBF value, and smoothed using a Gaussian filter at $16 \mathrm{~mm}$ FWHM. Analysis of the realignment parameters indicated that head movements of two of the participants exceeded 5 $\mathrm{mm}$ throughout the scan session. These two participants were, therefore, excluded from subsequent analysis to preclude activations due to movement artefact (Brett, Bloomfield, Brooks, Stein, \& Grasby, 1999). For the remaining nine participants, time (scan order) and head movement in the three planes of rotation and three dimensions of translation were set as confounding factors. Changes in blood flow between experimental conditions were estimated for each voxel using SPM 99b (provided by the Wellcome Department of Cognitive Neurology, London, UK). The resulting statistical images were warped into standardized stereotactic space and then further transformed to fit the Talairach and Tournoux (1988) atlas for the anatomical localization of activation peaks. The statistical threshold for reporting a peak as significant was set at $p<.05$, corrected for multiple comparisons across the entire brain volume. Because of our a priori predictions regarding activations occurring within the prefrontal cortex, an uncorrected threshold of $p<.001$ was used for predicted effects in this region (Lee et al., 2000; Worsley, Evans, Marrett, \& Neelin, 1992; Worsley et al., 1996).

\section{Acknowledgments}

We thank everyone at the Wolfson Brain Imaging Centre, especially Nahal Mavaddat, Steve Downey, Iona Kendall, Tim Donovan, Dylan Pritchard, and Gary Hawes. We are also very grateful to Kavitha Srinivas for allowing us to use her unfamiliar object stimuli, Mieke Verfaellie, Marion Kellenbach, and Wilma Koutstaal for discussions about design and theory, and Andy Calder, Matthew Brett, and Daniel Bor for software assistance and statistical advice.

Reprint requests should be sent to: Jon Simons, Department of Psychology, Harvard University, Rm 860 William James Hall, 33 Kirkland Street, Cambridge, MA 02138. Email: jss@wjh.harvard.edu.

\section{REFERENCES}

Baxendale, S. A. (1997). The role of the hippocampus in recognition memory. Neuropsychologia, 35, 591-598.

Brett, M., Bloomfield, P., Brooks, D., Stein, J., \& Grasby, P. M. (1999). Scan order effects in PET activation studies are caused by motion artefact. Neurolmage, 9, S56.

Bruce, V. (1982). Changing faces: Visual and non-visual coding processes in face recognition. British Journal of Psychology, 73, 105-116.

Buckner, R. L., \& Koutstaal, W. (1998). Functional neuroimaging studies of encoding, priming, and explicit memory retrieval. Proceedings of the National Academy of Sciences, U.S.A., 95, 891-898.

Buckner, R. L., Kelley, W. M., \& Petersen, S. E. (1999). Frontal cortex contributes to human memory formation. Nature Neuroscience, 2, 311-314.

Buckner, R. L., Koutstaal, W., Schacter, D. L., \& Rosen, B. R. (2000). Functional MRI evidence for a role of frontal and inferior temporal cortex in amodal components of priming. Brain, 123, 620-640.

Cabeza, R., \& Nyberg, L. (2000). Imaging cognition II: An em- 
pirical review of 275 PET and fMRI studies. Journal of Cognitive Neuroscience, 12, 1-47.

Cherry, S. R., Woods, R. P., Doshi, N. K., Banerjee, P. K., \& Mazziotta, J. C. (1995). Improved signal-to-noise in PET activation studies using switched paradigms. Journal of $\mathrm{Nu}$ clear Medicine, 36, 307-314.

Cohen, N. J., \& Eichenbaum, H. B. (1993). Memory, amnesia, and the hippocampal system. Cambridge: MIT Press.

Cohen, J. D., MacWhinney, B., Flatt, M., \& Provost, J. (1993). PsyScope: A new graphic interactive environment for designing psychology experiments. Behavioral Research Methods, Instruments and Computers, 25, 257-271.

Cooper, L. A., Schacter, D. L., Ballesteros, S., \& Moore, C. (1992). Priming and recognition of transformed three-dimensional objects: Effects of size and reflection. Journal of Experimental Psychology: Learning, Memory, and Cognition, 18, 43-57.

Dolan, R. J., \& Fletcher, P. C. (1997). Dissociating prefrontal and hippocampal function in episodic memory encoding. Nature, 388, 582-585.

Fletcher, P. C., Shallice, T., \& Dolan, R. J. (1998). The functional roles of prefrontal cortex in episodic memory: I. Encoding. Brain, 121, 1239-1248.

Fletcher, P. C., Shallice, T., Frith, C. D., Frackowiak, R. S. J., \& Dolan, R. J. (1998). The functional roles of prefrontal cortex in episodic memory: II. Retrieval. Brain, 121, 1249-1256.

Foundas, A. L., Daniels, S. K., \& Vasterling, J. J. (1998). Anomia: Case studies with lesion localisation. Neurocase, 4, 35-43.

Gabrieli, J. D. E., Brewer, J. B., Desmond, J. E., \& Glover, G. H. (1997). Separate neural bases of two fundamental memory processes in the human medial temporal lobe. Science, 276 , 264-266.

Gazzaniga, M. S., Bogen, J. E., \& Sperry, R. W. (1962). Some functional effects of sectioning the cerebral commissures in man. Proceedings of the National Academy of Sciences, U.S.A., 48, 1765-1769.

Gerlach, C., Law, I., Gade, A., \& Paulson, O. B. (1999). Perceptual differentiation and category effects in normal object recognition: A PET study. Brain, 122, 2159-2170.

Graham, K. S., Simons, J. S., Pratt, K. H., Patterson, K., \& Hodges, J. R. (2000). Insights from semantic dementia on the relationship between episodic and semantic memory. Neuropsychologia, 38, 313-324.

Hodges, J. R., Patterson, K., Oxbury, S., \& Funnell, E. (1992). Semantic dementia: Progressive fluent aphasia with temporal lobe atrophy. Brain, 115, 1783-1806.

Kanwisher, N., Woods, R. P., Iacoboni, M., \& Mazziotta, J. C. (1997). A locus in human extrastriate cortex for visual shape analysis. Journal of Cognitive Neuroscience, 9, 133-142.

Kelley, W. M., Miezin, F. M., McDermott, K. B., Buckner, R. L., Raichle, M. E., Cohen, N. J., Ollinger, J. M., Akbudak, E., Conturo, T. E., Snyder, A. Z., \& Petersen, S. E. (1998). Hemispheric specialization in human dorsal frontal cortex and medial temporal lobe for verbal and nonverbal memory encoding. Neuron, 20, 927-936.

Kelley, W. M., Buckner, R. L., Miezin, F. M., Cohen, N. J., Raichle, M. E., \& Petersen, S. E. (1999). Encoding of famous and nonfamous faces using fMRI. Society for Neuroscience Abstracts, 24, 760.

Kim, J. J., Andreasen, N. C., O'Leary, D. S., Wiser, A. K., Boles Ponto, L. L., Watkins, G. L., \& Hichwa, R. D. (1999). Direct comparison of the neural substrates of recognition memory for words and faces. Brain, 122, 1069-1083.

Klingberg, T., \& Roland, P. E. (1998). Right prefrontal activation during encoding, but not during retrieval, in a non-verbal paired-associates task. Cerebral Cortex, 8, 73-79.

Lee, A. C. H., Robbins, T. W., Pickard, J. D., \& Owen, A. M. (2000). Asymmetric frontal activation during episodic mem- ory: The effects of stimulus type on encoding and retrieval. Neuropsychologia, 38, 677-692.

Leveroni, C. L., Seidenberg, M., Mayer, A. R., Mead, L. A., Binder, J. R., \& Rao, S. M. (2000). Neural systems underlying the recognition of familiar and newly learned faces. Journal of Neuroscience, 20, 878-886.

McDermott, K. B., Buckner, R. L., Petersen, S. E., Kelley, W. M., \& Sanders, A. L. (1999). Set- and code-specific activation in the frontal cortex: An fMRI study of encoding and retrieval of faces and words. Journal of Cognitive Neuroscience, 11, 631-640.

Milner, B. (1970). Memory and the medial temporal regions of the brain. In K. H. Pribram \& D. E. Broadbent (Eds.), Biology of memory (pp. 29-50). London: Academic Press.

Milner, B. (1972). Disorders of learning and memory after temporal lobe lesions in man. Clinical Neurosurgery, 19, 421-446.

Milner, B., Petrides, M., \& Smith, M. L. (1985). Frontal lobes and the temporal organization of memory. Human Neurobiology, 4, 137-142.

Moscovitch, M., \& Winocur, G. (1995). Frontal lobes, memory, and aging. Annals of the New York Academy of Sciences, 769, 119-150.

Mummery, C. J., Patterson, K., Wise, R. J. S., Vandenberghe, R., Price, C. J., \& Hodges, J. R. (1999). Disrupted temporal lobe connections in semantic dementia. Brain, 122, 61-73.

Murtha, S., Chertkow, H., Beauregard, M., \& Evans, A. (1999). The neural substrate of picture naming. Journal of Cognitive Neuroscience, 11, 399-423.

Nyberg, L., Cabeza, R., \& Tulving, E. (1996). PET studies of encoding and retrieval: The HERA model. Psychonomic Bulletin and Review, 3, 135-148.

Nyberg, L., McIntosh, A. R., Houle, S., Nilsson, L.-G., \& Tulving, E. (1996). Activation of medial temporal structures during episodic memory retrieval. Nature, 380, 715-717.

Owen, A. M., Milner, B., Petrides, M., \& Evans, A. C. (1996). Memory for object features versus memory for object location: A positron-emission tomography study of encoding and retrieval processes. Proceedings of the National Academy of Sciences, U.S.A., 93, 9212-9217.

Paivio, A., \& Csapo, K. (1973). Picture superiority in free recall: Imagery or dual coding? Cognitive Psychology, 5, 176-206.

Patterson, K., \& Hodges, J. R. (2000). Semantic dementia: One window on the structure and organisation of semantic memory. In L. S. Cermak (Ed.), Handbook of neuropsychology, 2nd edition, Vol. 2: Memory and its disorders (pp. 313-333). Amsterdam: Elsevier.

Price, C. J. (1998). The functional anatomy of word comprehension and production. Trends in Cognitive Sciences, 2, 281-288.

Price, C. J., Wise, R. J. S., Warburton, E. A., Moore, C. J., Howard, D., Patterson, K., Frackowiak, R. S. J., \& Friston, K. J. (1996). Hearing and saying: The functional neuro-anatomy of auditory word processing. Brain, 119, 919-931.

Schacter, D. L., \& Wagner, A. D. (1999). Medial temporal lobe activations in fMRI and PET studies of episodic encoding and retrieval. Hippocampus, 9, 7-24.

Schacter, D. L., Reiman, E. M., Uecker, A., Polster, M. R., Yung, L. S., \& Cooper, L. A. (1995). Brain regions associated with retrieval of structurally coherent visual information. Nature, 376, 587-590.

Schacter, D. L., Alpert, N. M., Savage, C. R., Rauch, S. L., \& Albert, M. S. (1996). Conscious recollection and the human hippocampal formation: Evidence from positron emission tomography. Proceedings of the National Academy of Sciences, U.S.A., 93, 321-325.

Schacter, D. L., Uecker, A., Reiman, E. M., Yun, L. S., Bandy, D., 
Chen, K. W., Cooper, L. A., \& Curran, T. (1997). Effects of size and orientation change on hippocampal activation during episodic recognition: A PET study. NeuroReport, 8, 3993-3998.

Shallice, T., Fletcher, P., Frith, C. D., Grasby, P., Frackowiak, R. S. J., \& Dolan, R. J. (1994). Brain regions associated with acquisition and retrieval of verbal episodic memory. Nature, 368, 633-635.

Shimamura, A. P. (1995). Memory and frontal lobe function. In M. S. Gazzaniga (Ed.), The cognitive neurosciences, 1st ed., (pp. 803-813). Cambridge: MIT Press.

Simons, J. S., Graham, K. S., \& Hodges, J. R. (1999). What does semantic dementia reveal about the functional role of the perirhinal cortex? Trends in Cognitive Sciences, 3, 248-249.

Simons, J. S., Graham, K. S., Galton, C. J., Patterson, K., \& Hodges, J. R. (2001). Semantic knowledge and episodic memory for faces in semantic dementia. Neuropsychology, 15, 101-114.

Squire, L. R. (1987). Memory and brain. Oxford: Oxford University Press.

Squire, L. R. (1992). Memory and the hippocampus: A synthesis from findings with rats, monkeys, and humans. Psychological Review, 99, 195-231.

Srinivas, K. (1995). Representation of rotated objects in explicit and implicit memory. Journal of Experimental Psychology: Learning, Memory, and Cognition, 21, 1019-1036.

Srinivas, K., Breedin, S. D., Coslett, H. B., \& Saffran, E. M. (1997). Intact perceptual priming in a patient with damage to the anterior inferior temporal lobes. Journal of Cognitive Neuroscience, 9, 490-511.

Talairach, J., \& Tournoux, P. (1988). Co-planar stereotaxic atlas of the buman brain. Stuttgart, Germany: Thieme.

Thompson-Schill, S. L., D'Esposito, M., Aguirre, G. K., \& Farah, M. J. (1997). Role of left inferior prefrontal cortex in retrieval of semantic knowledge: A reevaluation. Proceedings of the National Academy of Sciences, U.S.A., 94, 1479214797.

Tulving, E., \& Markowitsch, H. J. (1998). Episodic and declarative memory: Role of the hippocampus. Hippocampus, 8, 198-204.

Tulving, E., Kapur, S., Craik, F. I. M., Moscovitch, M., \& Houle, S. (1994). Hemispheric encoding/retrieval asymmetry in episodic memory: Positron emission tomography findings. Proceedings of the National Academy of Sciences, U.S.A., 2016-2020.

Tulving, E., Markowitsch, H. J., Craik, F. I. M., Habib, R., \& Houle, S. (1996). Novelty and familiarity activations in PET studies of memory encoding and retrieval. Cerebral Cortex, 6, 71-79.

Vandenberghe, R., Price, C. J., Wise, R. J. S., Josephs, O., \& Frackowiak, R. S. J. (1996). Functional anatomy of a common semantic system for words and pictures. Nature, 383, 254256.

Vargha-Khadem, F., Gadian, D. G., Watkins, K. E., Connelly, A., Van Paesschen, W., \& Mishkin, M. (1997). Differential effects of early hippocampal pathology on episodic and semantic memory. Science, 277, 376-380.

Wagner, A. D., Desmond, J. E., Glover, G. H., \& Gabrieli, J. D. E. (1998). Prefrontal cortex and recognition memory: Functional-MRI evidence for context-dependent retrieval processes. Brain, 121, 1985-2002.

Wagner, A. D., Poldrack, R. A., Eldridge, L. L., Desmond, J. E., Glover, G. H., \& Gabrieli, J. D. E. (1998). Material-specific lateralization of prefrontal activation during episodic encoding and retrieval. NeuroReport, 9, 3711-3717.

Warburton, E., Wise, R. J. S., Price, C. J., Weiller, C., Hadar, U., Ramsay, S., \& Frackowiak, R. S. J. (1996). Noun and verb retrieval by normal subjects: Studies with PET. Brain, 119, 159-179.

Warrington, E. K. (Ed.). (1984). Recognition memory test. Windsor, UK: NFER Nelson.

Warrington, E. K., \& James, M. (1967). An experimental investigation of facial recognition in patients with unilateral cerebral lesions. Cortex, 3, 317-326.

Wheeler, M. A., Stuss, D. T., \& Tulving, E. (1995). Frontal lobe damage produces episodic memory impairment. Journal of the International Neuropsychological Society, 1, 525-536.

Worsley, K. J., Evans, A. C., Marrett, S., \& Neelin, P. (1992). Determining the number of statistically significant areas of activation in subtracted activation studies from PET. Journal of Cerebral Blood Flow and Metabolism, 12, 900-918.

Worsley, K. J., Marrett, S., Neelin, P., Vandal, A. C., Friston, K. J., \& Evans, A. C. (1996). A unified statistical approach for determining significant signals in images of cerebral activation. Human Brain Mapping, 4, 58-73. 